\title{
Dissection of AI Job Advertisements: A Text Mining-based Analysis of Employee Skills in the Disciplines Computer Vision and Natural Language Processing
}

\author{
Henrik Kortum \\ DFKI, \\ Osnabrück, Germany \\ henrik.kortum@dfki.de
}

\author{
Jonas Rebstadt \\ DFKI, \\ Osnabrück, Germany \\ jonas.rebstadt@dfki.de
}

\author{
Oliver Thomas \\ DFKI, \\ Osnabrück, Germany \\ oliver.thomas@dfki.de
}

\begin{abstract}
Human capital is a well discussed topic in information system research. In order for companies to develop and use IT artifacts, they need specialized employees. This is especially the case when complex technologies, such as artificial intelligence, are used. Two major fields of artificial intelligence are computer vision (CV) and natural language processing (NLP). In this paper skills required for $C V$ and $N L P$ specialists are analyzed and compared from a job market perspective. For this purpose, we utilize a text mining-based analysis pipeline to dissect job advertisements for artificial intelligence. In concrete, job advertisements of both sub-disciplines were crawled from a large international online job platform and analyzed using named entity recognition and term vectors. It could be shown that skills required differ between the two job profiles. There is no general requirement profile of an artificial intelligence specialist, and it requires a differentiated consideration.
\end{abstract}

\section{Introduction}

Two major application fields of artificial intelligence (AI) are computer vision (CV) and natural language processing (NLP) [1]. CV technologies are successfully used in a variety of scenarios to improve processes e.g. in manufacturing [2, 3, 4], medicine [5, 6] or retail [7]. In addition, $\mathrm{CV}$ is also integrated into products such as smartphones for facial detection [8]. A wide variety of methods as classification, object detection and semantic segmentation are used [9]. Since 2012, there has been increased use of deep learning methods to solve CV tasks. Deep neural networks with multiple hidden layers are trained to solve specific problems [10]. The breakthrough for deep learning CV applications was triggered in 2012 by the winning team of the image net classification challenge [11]. With this breakthrough, the achievable accuracies could be boosted and previously impossible use cases could be realized [1] Next to CV, NLP is a widespread field of AI applications in companies [12]. NLP is e.g. used for automatic classification of documents [13] and supports further automation of processes. This is particularly the case in finance-related areas [14]. In addition NLP can also be applied to automate communication, e.g. in chatbots [15]. The use of conversational agents is mainly due to the progress in NLP [16]. Conversational agents are used e.g. by companies for initial customer contact or for the general improvement of customer service [17]. One field with great relevance is sentiment analysis [18]. NLP also plays a key role in the implementation of virtual personal assistants such as Alexa [19] and Siri [20] or enables automated translation of texts, e.g. DeepL or Google Translate. The application of CV technologies as well as NLP is becoming more and more complex and the field is developing very dynamically, both in theory and in practice. In 2019 alone, there were 2,887 publications on the IEEE platform on the subject of CV [21]. Consequently, the topics are also evolving from a practical perspective, which is reflected by a sharp increase in demand for AI skills in job advertisements over the past decade [22]. For companies to use CV and NLP, they need highly qualified personnel. Due to the dynamic development, the requirements for $\mathrm{CV}$ and NLP specialists are constantly changing. While for $\mathrm{AI}$ in general the requirements for employees have been investigated [23], the requirements that arise for the two largest specialized fields, CV and NLP, remain largely unconsidered. As application fields become more specialized and complex, the question arises whether a general consideration of employee skills in the context of AI is sufficient, or whether a more differentiated consideration of this growing heterogeneity should be taken. This results in following research questions (RQ): RQ1: Do CV job advertisements show significant differences to NLP job advertisements regarding required skills?

RQ2: What are the required skills for CV and NLP specialists from a job market perspective and what are the concrete differences?

To investigate the RQs, we analyzed 1,159 job postings from the online job platform Indeed using text mining and data analysis methods. First, we describe the relevant background in Section 2 followed by Section 3 
where we outline our research approach. In concrete, we used named entity recognition (NER) and term frequency - inverse document frequency (TF-IDF) vector representation to investigate similarities between different job advertisements. The results are described, categorized, and compared for CV and NLP in Section 4. We critically discuss our results in Section 5 and conclude the paper with an outlook in Section 6.

\section{Background}

In information systems research, the field of human capital plays a crucial role [23, 24, 25, 26]. Human capital describes the knowledge, skills, know-how and other acquired personal characteristics that are considered useful in creating value [27]. Employee skills that are relevant to perform a specific task can be classified as "task-related" human capital [28]. In this sense, the skills identified in our work are related to CV and NLP. Since these skills are rare, time-consuming to acquire and often associated with a high level of education, appropriately skilled employees are rare resources whose management by HR has the potential to create competitive advantage [29]. Besides technical skills, soft skills also play a crucial role on the job market in general [30] and especially in IT-related professions [31]. The identification and formalization of such relevant skills is in turn an essential element in the creation of job profiles [32]. Due to changing and emerging technologies, new job requirements will arise in the future, even completely new job profiles will emerge and some will disappear $[24,33]$. This leads to the need for practitioners and academics to understand which skills in demand are increasing [24]. Regarding jobs related to AI, there is a tendency for new requirements and job profiles to emerge. Data is increasingly becoming one of the most relevant resources for companies [12]. In this context, data analytics and AI play an important role in the valueadding transformation of raw data. The development of such value-adding AI-based systems is a very complex task that requires highly skilled personnel and various qualifications [12]. A lack of specialized experts in this field already exists and the absence of qualified personnel is a major challenge for companies [34]. But among other resources, skilled personnel contribute to the AI readiness of companies and the successful implementation of AI projects [35]. For companies to be able to employ trained specialists or to train them further, a detailed description of the skills in demand is required. Anton et al. [23] provided a detailed list of human AI competencies. Among the most important competencies are technical and management skills. The level of detail within the competence classes is already very pronounced, but there is no detail at the level of sub-disciplines, such as CV or NLP. The analysis and study of skills in demand on the job market is associated with a high level of effort. There are various data sources and a large number of available job advertisements.

\section{Research Approach}

\subsection{Method Description}

We use text mining - a method closely related to NLP - to analyze large amounts of job advertisements in a semi-automated way. NLP methods are already used in the scientific context to extract keywords from texts [36], to generate summaries [37] or to find research trends [21]. Also regarding the evaluation and analysis of skills, which are in demand on the job market, first approaches for the evaluation of job advertisements with NLP can be found in the literature [23].

\subsection{Data Collection}

To dissect job profiles of CV engineers and NLP specialists, advertisements from Indeed were collected in the period from 29th September to 5th October 2020 for CV and on $7^{\text {th }}$ November 2020 for NLP. Indeed was chosen because it is one of the largest job platforms and considers job advertisements from all over the world. Moreover, Indeed publishes them as text, which allows for easy crawling of the content. CV job advertisements were considered whose title contains the keywords "computer" and "vision", to ensure that only relevant hits are considered. The search term "title:(computer vision)" was used on 56 top level domains. The search identified 785 job advertisements. For NLP the search term "title:((natural language understanding) or (natural language processing) or (NLP))" was used and 374 job advertisements were identified. To extract the content from the websites, octoparse (https:/www.octoparse.com/) was used. We extracted the job title, the location and the advertisement body. For further analysis only the job advertisements in English were taken into account and therefore the corpus for the further analysis consists of 591 job advertisements for CV and of 291 for NLP. The job advertisements used are spread over more than 30 countries. The countries with the most job openings in CV are USA, Israel and Germany and in NLP these are USA, United Kingdom and India (See Appendix, for review only).

\subsection{Text Mining Pipeline}

Our text mining pipeline consists of 5 steps: preprocessing, named entity recognition, human validation, processing and data analysis. In Figure 1 the pipeline is visualized. 


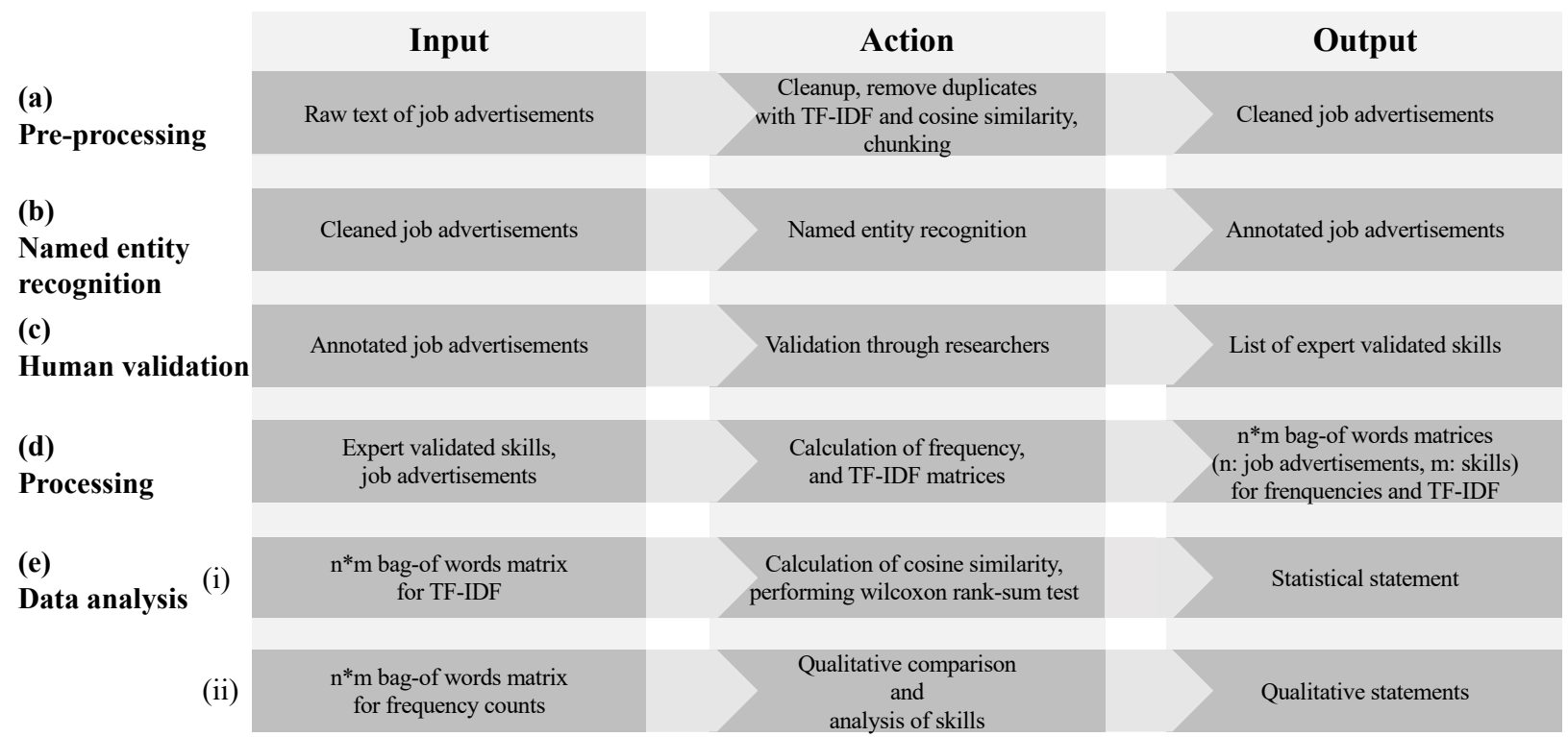

Figure 1. Text mining pipeline for analyzing job advertisements

(a) Pre-processing: Input for pre-processing was the raw text string extracted from the job advertisements. At first, all characters were transformed into lower case. Subsequently, the removal of duplicate job advertisements was carried out. There are numerous methods for the recognition of text duplicates, one widely used variant is based on learnable vector-space similarity [e.g. 38, 39]. In our case, the raw text string was transformed into the broadly used TF-IDF vectors to determine the mutual cosine similarity between each of them. The TFIDF of a term $t$ in a document $d$ is defined as the product of the frequency of occurrence (TF) of the term in the specific document and the inverse document frequency of the word (IDF) across all documents in the corpus. There are different implementations of the TF-IDF algorithm, which differ slightly in form. We used the function TfidfVectorizer of the Python library sklearn. For the calculation of the cosine similarity, we also made use of sklearn, utilizing the pairwise.cosine_similarity function. All pairs with a similarity of more than 0.99 were treated as duplicates and one entry was removed randomly, resulting in a set of 733 unique documents. After removing the duplicates, the TF-IDF vectors were removed, and the cleaned text strings were used in the further workflow. Since the service we subsequently used has the restriction of only being able to process batches of 5 documents, the corpus was first broken down into corresponding batches of 5 . (b) Named entity recognition (NER): Since our goal is to dissect job advertisements with regard to the skills required, the first step is to identify relevant terms representing a particular skill. The identification of certain term classes within a text corpus is a NER task [40, e.g.
41] for which in general supervised learning approaches are suitable. However, these methods rely on large amounts of labeled training data, and since there was a lack of suitable data to build a dedicated training base for the NER part, we decided to use a cloud service to support us in the process of skill identification. In concrete the Microsoft Azure Analytics Service was used to retrieve terms from the corpus referring to skill entities. It was chosen because it has already been trained on a large number of documents and explicitly supports the classification of skill entities. Input for the service are the pre-processed and cleaned job advertisement text strings, consisting of a combination of 13,201 unique terms. The service output is a JSON string, which contains mapped word - entity pairs as attributes. For further processing all non-skill entities were filtered out, resulting in 3,712 skill terms. (c) Human validation: The results from step (b) were then evaluated by two researchers. Semantically similar terms for a skill, such as "nlp" and "natural language processing", were combined into one term. The researchers were supported during the identification of such synonyms by an artificial neural network for the calculation of vector-based word similarities. The method used is described in detail in [21]. In addition, we removed terms that were not skills in the sense of our analysis. Of the initial 3,712 terms identified as skills, 523 unique terms remained after validation. In the next step, the skills were allocated into seven more abstract categories. The skills were manually assigned to one of the following categories: AI, programming, programming language, general skills, domain, soft skills, and qualification. This enables a detailed evaluation within the upper categories. 
(d) Processing For further investigation, the raw text was transformed so that all non-skill words were treated as stop words and removed from it. As a result, we received a sequence consisting of the skills from the job advertisement for each document. Based on these sequences, bag-of-words frequency and TF-IDF matrices were calculated. This time the TF-IDF vectors were used to provide more differentiated statements about the relevance of a term for a single document. In contrast to a simple word count, which only contains information on how often a certain term is contained in a document, the TF-IDF score allows conclusions to be drawn about the relevance of the term within a document and within the entire corpus [42]. A high value indicates a high relevance of the word in the respective document, a low value indicates a lower relevance. Later, we will use this characteristic in the data analysis to automatically calculate similarities between job advertisements. This way we generated a $n \times m$ matrix, where $n$ is the number of input documents and $m$ is the number of words in the input vocabulary. The values inside the matrix are the TF-IDF calculated scores. (e) Data analysis: Our data analysis consists of two parts. In (i) we analyzed whether there is a significant difference between the relevant terms and CV job advertisements in comparison to NLP job advertisements. In (ii) a qualitative detailed investigation is performed at the level of skill classes, down to the level of concrete skills. (i) To investigate the similarity of the two sample text corupuses for NLP and $\mathrm{CV}$, with regard to the skills contained in the respective job advertisements, we again made use of the calculation cosine similarity, this time based on the TFIDF matrix for skills, we received from step (d). The results are visualized by distribution and heatmap plots. To test the significance of the results, a Wilcoxon ranksum test [43] was performed. (ii) Within the individual top categories, the skills have been evaluated. In addition to simple mentions (as e.g. in Figure 3) it was further evaluated in how many job advertisements a specific skill was mentioned (double mentions within a job advertisement are not counted twice). Afterwards the relative share is calculated in order to establish comparability between CV and NLP job advertisements.

\subsection{Triangulation of Results}

Finally, the results obtained through our text mining pipeline are compared with the current state of the literature in order to evaluate them through scientific findings. In doing so, we try to relate the insights gained through the analysis of the job advertisement to aspects discussed in the literature.

\section{Results}

\subsection{Similarity Analysis}

Through our analysis, we were able to show that $\mathrm{CV}$ job advertisements have an average class-internal cosine similarity of 0.11 , while NLP publications have an average similarity of 0.14 . The average similarity of an NLP job posting to a CV posting is 0.07 . This finding indicates, that there is an overall dissimilarity between $\mathrm{CV}$ and NLP job postings. A wilcoxon rank-sum test [43] was performed to statistically validate these finding. It was shown that at all significance levels, the null hypothesis that, for randomly selected similarity values $S_{\mathrm{CV}: \mathrm{CV}}$ and $S_{\mathrm{CV}: \mathrm{NLP}}$, the probability of $S_{\mathrm{CV}: \mathrm{CV}}$ being greater than $S_{\mathrm{CV}: \mathrm{NLP}}$ is equal to the probability of $S_{\mathrm{CV} \text { :NLP }}$ being greater than $S_{\mathrm{CV}: \mathrm{CV}}$, can be rejected. The same applies to the random comparison of two $S_{\mathrm{NLP} \text { NLP }}$ and

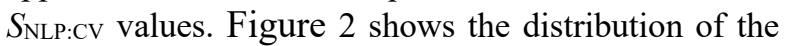
mutual cosine similarity for the pairs of job advertisements: CV:CV, NLP:NLP, CV:NLP and NLP:CV. Whereby the last two are symetrical to each other and therefore identical. The results shows a larger spread of the similarity distribution for the intraclass comparisons $\mathrm{CV}: \mathrm{CV}$ and NLP:NLP than for the interclass comparison CV:NLP. The similarity scores for CV:NLP accumulate at a lower level, indicating a greater dissimilarity of NLP and CV job postings. Furthermore, it can be stated that NLP job descriptions are fundamentally more similar to each other than $\mathrm{CV}$ job descriptions are to each other. Now that we answered RQ1 and it has been established that there is a significant difference between NLP and CV job advertisements in terms of skills, the following section provides an expert-based evaluation of these findings at the level of concrete skills.

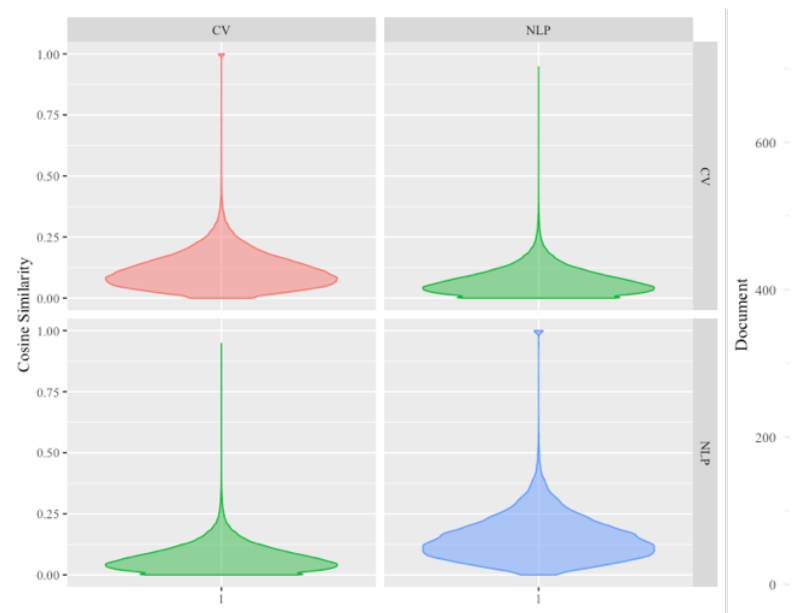

Figure 2. Visualization of the cosine similarity of document pairs 


\subsection{Content Analysis}

In the following, the differences and similarities between the two categories of job advertisements are examined in detail. Our analysis takes into account both task-specific skills like AI and IT skills, along with soft skills, since a combination of both types is essential for the

profession

[44].

\subsubsection{Specified AI Skills}

The evaluation of job advertisements has shown the explicit necessity of skills and knowledge around AI, both for NLP specialists and CV engineers. The two job profiles clearly overlap here. Skills frequently mentioned in relation to AI include e.g. deep learning, machine learning and data science. In addition to knowledge of statistics and mathematics, the understanding of different learning approaches (unsupervised, reinforcement, supervised, transfer learning) as well as different tasks like classification, clustering, pattern recognition, regression or anomaly detection is required. Skills related to data, such as data analysis or data preprocessing, are also frequently listed. Not only the development of models is required, but also the design of algorithms. This shows that extensive basic knowledge and specialized AI skills are frequently mentioned requirements. In addition to general AI skills, expert knowledge in CV and NLP is also required. In this aspect the two job profiles differ. For CV engineers, e.g. object detection, segmentation and 3D graphics skills are required. In contrast, for NLP specialists, skills like sentiment analysis, speech recognition and information extraction are required. Figure 3 provides an overview of the general and specific requirements related to AI for the job profiles of CV and NLP specialists.

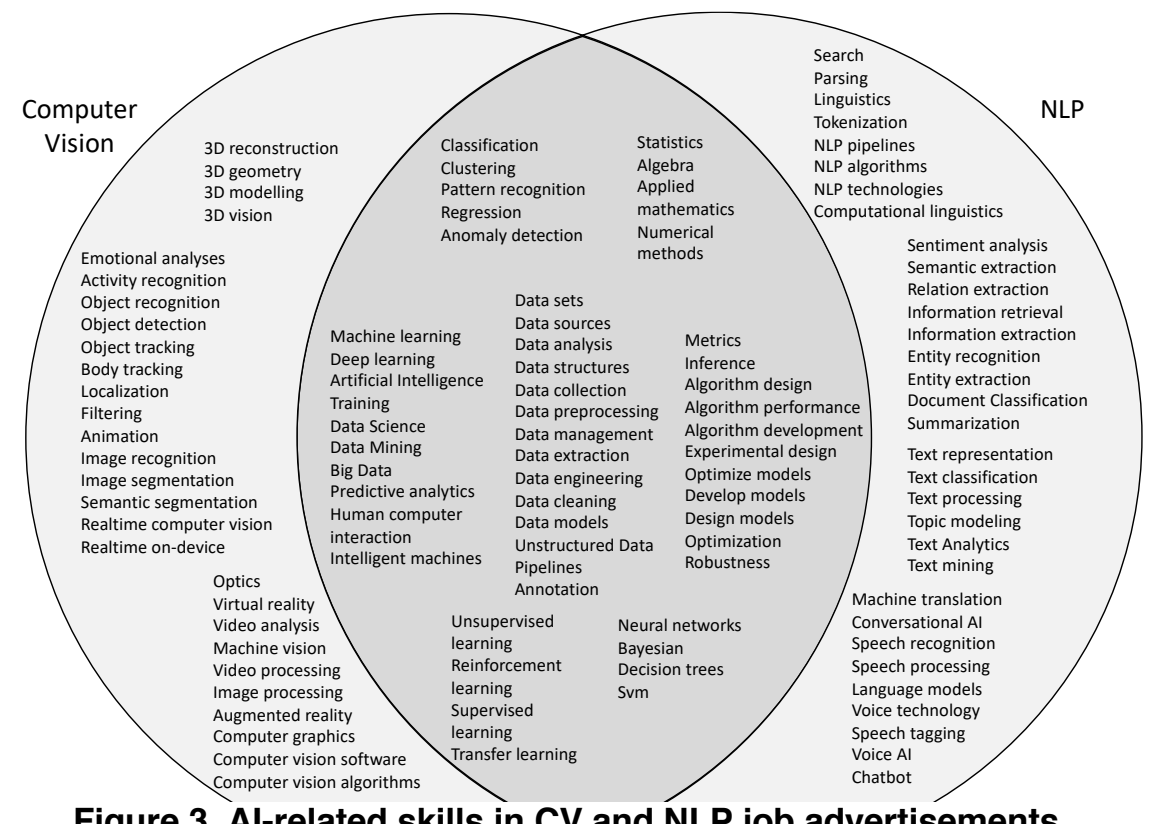

Figure 3. Al-related skills in CV and NLP job advertisements

\subsubsection{Required IT Skills}

Both the NLP and CV job advertisements call for IT skills. This includes, for example, general development and software engineering skills, methods, and best practices. The most frequently mentioned general skills related to IT and programming are listed below: General: Software development, engineering, implementation, design, architecture, Professional development, Maintenance, Computer engineering. Methods: DevOps, Scrum, Continuous integration, Systems engineering, Agile software development. Best Practices: Coding, Debugging, Prototyping, Unit testing, Version control, Code review, Containerization, Parallel computing, Distributed computing, Cloud computing. Of particular interest is the analysis of the required programming languages. Both, in CV and NLP job advertisements Python is the most frequently mentioned programming language. It is especially noticeable in $\mathrm{CV}$, that hardware-related programming languages, such as $\mathrm{C}++$, are used much more frequently than in NLP advertisements. Therefore, script languages like Python and R are mentioned more often in NLP advertisements. E.g., Python is mentioned in $82 \%$ of NLP and only in $55 \%$ of the CV advertisements. Figure 4 shows the programming languages and their frequency of nomination. 


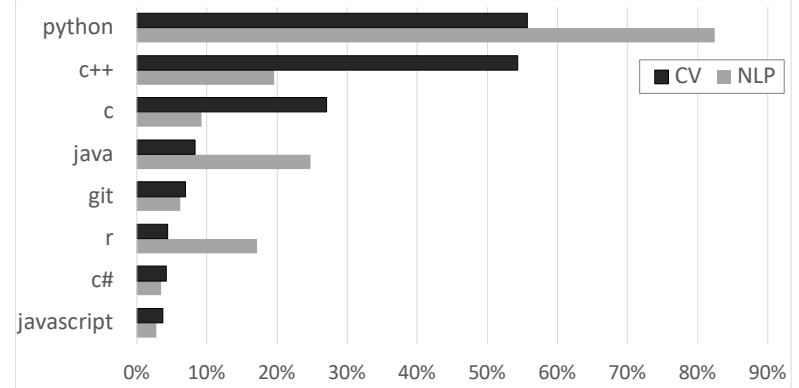

Figure 4. Most mentioned programming languages

In addition to the skills associated with programming, more general IT skills are also required. Engineering skills are required in both CV (43\%) and NLP $(33 \%)$. It is noticeable that in CV job advertisements hardware related skills plays a greater role. This is illustrated by the frequent use of the terms hardware (16\%), robotics (15\%) and IoT (7\%). These skills only play a minor role in NLP job advertisements. On the other hand, business skills are sought much more frequently in NLP (68\%) advertisements. Figure 5 gives an overview of the top 10 general IT skills for CV and NLP jobs.

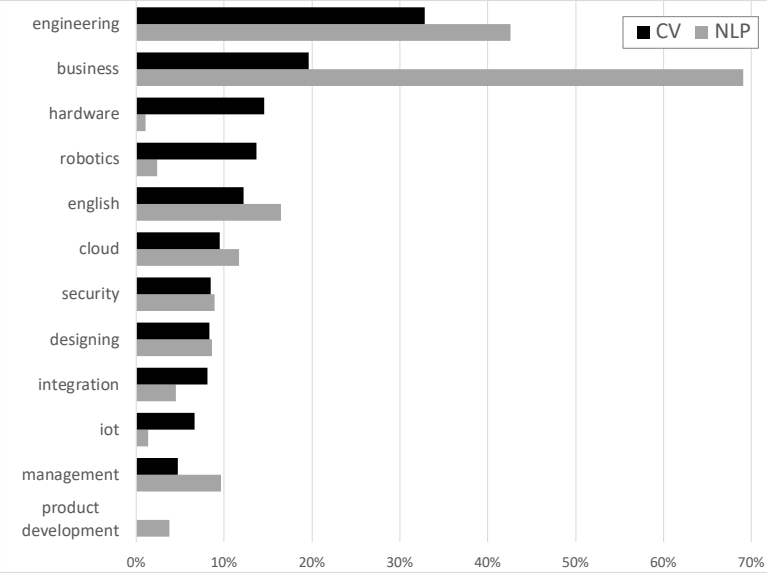

Figure 5. most mentioned general IT skills

\subsubsection{Required Soft Skills}

For the classification as "soft skills" we follow the definition of Weber et al. [45] according to which soft skills subsume people/relationship skills, communication skills, management/organization skills or cognitive skills [45]. An analysis of the soft skills required shows no major differences between NLP and CV (see Figure 6). For both specialists, the ability to collaborate is the most frequently required skill. Correspondingly, the second most frequently required skills are communication skills. In addition, the requirement for interpersonal skills is also found in both areas. In contrast to these cooperative skills, however, there is also an explicit demand for independent working methods and self-motivation. Other soft skills required are creativity, passion and a sense of responsibility.

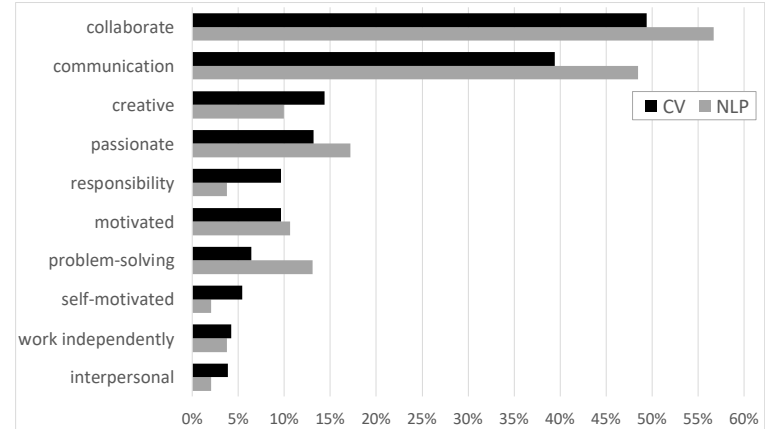

Figure 6. Most mentioned soft skills

\subsubsection{Qualifications}

In order to investigate which specific academic degrees are specified in the job advertisements, it was analyzed in which job advertisements $\mathrm{PhD}$, bachelor or master or a combination of these keywords occur. In $50 \%$ of the job advertisements of CV and NLP none of these keywords were present. In the others either one or a combination of these keywords were found. In concrete, $21 \%$ of $\mathrm{CV}$ job postings contain the keyword $\mathrm{PhD}$, whereas only $16 \%$ of NLP job postings contain the keyword PhD. These job advertisements then do not contain the keywords master or bachelor. Combinations of academic degrees were also considered. For example, the keywords master and $\mathrm{PhD}$ appear in $5 \%$ of $\mathrm{CV}$ and $8 \%$ of NLP job advertisements.

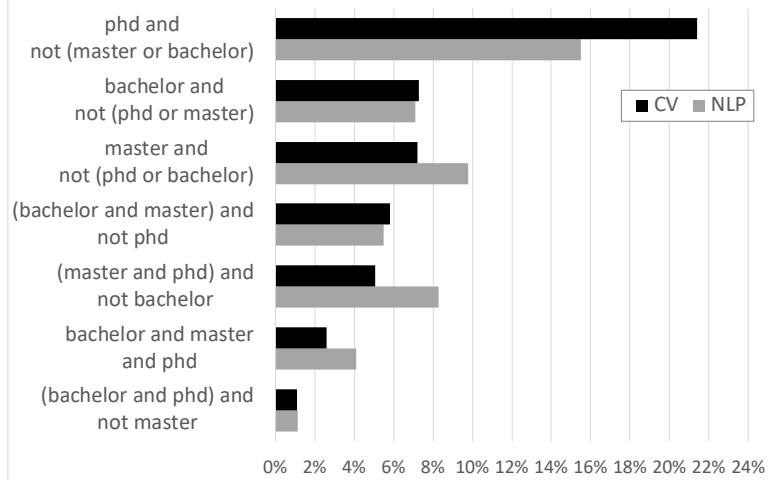

Figure 7. Mentioned academic degrees 


\subsection{Triangulation of Results with current Lit- erature}

The similarities and differences make sense from a subject-specific perspective. For example, it is comprehensible that for CV and NLP general AI knowledge is required, e.g., in the area of deep learning. The state of the art in both CV and NLP is based on these technologies and the significant successes and advances in recent years can even be attributed to them [1]. For example the latest NLP methods like BERT [46] and Elmo [47] are based on deep learning technologies, as well as object detection methods like Yolo [48]. The methodological foundations must therefore be mastered by both $\mathrm{CV}$ and NLP specialists. Differences arise in the various types of application of the methods specific for $\mathrm{CV}$ and NLP. The difference in required domain expertise in $\mathrm{CV}$ and NLP job advertisements could result from the potential offered by the technologies used in these areas. The potential of CV applications is particularly evident in applications in the health $[6,49,50]$, manufacturing [2, 3, 4], logistics, automotive [51] and retail [7] domains. Great potentials of NLP applications are in finance [14], health $[6,52]$ and legal [53]. The strong demand for experience in the programming language Python in NLP job advertisements can be attributed to the extensive library support of Python for NLP tasks. Python supports the leading deep learning libraries: Tensorflow, Pytorch or Keras, as well as many NLP specific tools like NLTK or Gensim, which are very popular in the NLP area [54]. Python is also a leading programming language for CV tasks because there is an extensive library support, e.g for Tensorflow. Nevertheless, especially for $\mathrm{CV}$ tasks, the computing time and inference time in applications is of high relevance [48]. With programming languages that are closer to hardware, like $\mathrm{C}++$ and $\mathrm{C}$, a higher performance can be achieved [55]. Furthermore, in many cases hardware integration is also necessary for $\mathrm{CV}$ tasks [56]. Both professions require a high level of education, so that employees from both disciplines can be considered a potential strategic resource [29]. Although the survey did not reveal any major differences between CV and NLP specialists, a deeper look at the specific soft skills offers interesting insights. It is particularly interesting that the two most frequently requested skills are collaboration and communication skills, and that they are requested in almost every second job advertisement. This confirms that AI specialists in companies need more than just analytical and IT skills and that a considerable part of their work is based on collaboration and communication [57]. In contrast to Anton et al. [23], however, we could not confirm a high relevance of management skill for the investigated sub-areas. A reason for this could be that $\mathrm{CV}$ and
NLP experts are technically in-depth professions that are more distinct from AI management professions.

\section{Discussion}

\subsection{Implications}

From a theoretical perspective, (T1) we provide the information systems community with an extensive collection of CV- and NLP-related skills. With this we complement the collection of necessary AI-related skills, e.g. the collection in Anton et al. [23], and show that a differentiated perspective makes sense. (T2) We could prove that there is a significant difference between the job profiles of CV engineers and NLP specialists in terms of required skills. Consequently, for further discussions from a theoretical perspective about human AI skills, a more differentiated approach should be chosen to take the specialized fields into account. (T3) The keyword collection of AI terms provided by us is also a promising starting point for the differentiated labeling of skill-related entities in text documents. In this way, our work helps other researchers to solve the problem of missing NER models for skill identification, to which we were also confronted. (T4) The text mining pipeline we present can also be applied as a method in other research areas. However, its success is largely dependent on the existence of a NER algorithm that can recognize the desired class of entities, as in our case, skills. In addition, a sufficient number of text documents must be available as data basis. (T5) From an HR research perspective, our work offers an empirical contribution to the body of knowledge by providing a differentiated assessment of task-related skills as well as general IT and soft skills drawn from the two largest AI subfields. (T6) In doing so, we also contribute to human capital theory by analyzing in detail the strategic resource of $\mathrm{CV}$ and NLP experts. Implications also arise for practitioners. (P1) NER proofed usefulness to extract relevant skills from job advertisements. This has enabled us to efficiently analyze a large number of job advertisements and to create general skillsets for CV engineers and NLP specialists. These general skillsets can support companies in the creation of CV and NLP job advertisements. In particular, we address companies for which $\mathrm{CV}$ and NLP are new and promising areas. We assume that these companies often do not know in detail which skills - especially technical skills - are required to fulfil $\mathrm{CV}$ and NLP related tasks. (P2) In the future, the methodology can also be used to automatically evaluate other job advertisements and serve as a template for job advertisements in other areas, because NER is a supervised approach and a setting of parameters is not necessary when predicting entities, like it is the case e.g. in topic model- 
ing methods used in Anton et. al [23] to analyze job advertisements. (P3) We could show that there are different requirements regarding skills in the two subcategories $\mathrm{CV}$ and NLP. If a company is now looking for an AI expert, the job advertisement should already indicate which AI application and methods need to be used. (P4) Since a lack of AI competencies is often mentioned as a barrier for the adoption of AI technologies [34, 58], our results can serve as a summary for companies to proof their AI readiness in specific to the methods $\mathrm{CV}$ and NLP. (P5) In addition, companies can use our list to manage their further education to develop personalized programs including technical skills as well as the required soft skills. (P6) Companies may even extend these ideas towards comprehensive training programs in order to counteract the persistent shortage of skilled workers. This is especially exciting since many of the job postings call for collaboration and communication skills, which are only a very limited part of many AI expert trainings. (P7) Going one step further, our findings can also be used to feed back into the education sector. Universities and other educational institutions may use the practical requirements obtained from job advertisements to enhance their curricula. (P8) Overall, the presented approach and the raised knowledge reduces the risk of decoupled HR and specialist departments by increasing the knowledge base in the HR departments and the forementioned automation options in personnel acquisition. (P9) But our evaluations are not only interesting for companies. Due to the extremely differentiated evaluation, applicants for CV and NLP jobs can compare their skills with the ones required in the advertisement.

\subsection{Limitations}

We want to discuss two main limitations regarding the text corpus and the method. First, it should be mentioned with regard to the text corpus that there are limitations about the time dimension, since the sample on which the analysis is based is only a snapshot and thus does not allow statements about the continuity and robustness of the findings over time. In addition, the text corpus contains only English-language job advertisements. This means that job advertisements from nonEnglish speaking countries that were only available in the local language have not been included in the analysis. Furthermore, we only used one job portal for the data collection. Although Indeed is one of the largest platforms for job advertisements, it cannot be guaranteed that the sample allows a statement about the population. A statement about the validity of the job advertisements is also difficult since there is no information available whether the job advertisement was written by a specialist department or a human resources department. It should be noted, however, that a job advertisement reflects the demand of the job market, which is to be examined in this paper. Nevertheless, by analysing the two samples with a wilcoxon rank-sum test for all significance levels, we were able to prove that NLP job advertisements differ significantly from $\mathrm{CV}$ job advertisements regarding the relevant skills. In addition, the results could be expertly validated at the level of individual skill categories and skills that are in concrete demand. The NER and TF-IDF vector representation tools used for the analysis are suitable tools for the quantitative evaluation of large unstructured text quantities. These methods, however, are not without flaws. For example, there is a risk that the NER may not have all skills identified that could have been potentially relevant for the study. Sometimes, for example, paraphrases of a word such as "strong academic background" could be used as a synonym for a degree. However, even a purely human analysis of the job advertisement would not have ensured error-free identification, which is why we decided to use NLP methods as machine support. In addition, it is to be mentioned that the labeled skills were manually checked by two researchers.

\section{Conclusion and Outlook}

In this paper we have examined and compared the requirements for $\mathrm{CV}$ engineers and NLP specialists from the perspective of the job market. We crawled job postings from Indeed and analyzed them using a text mining pipeline and manual processing to identify the relevant skills. With regard to RQ1, we could prove that the required skills in NLP and CV job advertisements differ significantly. To answer RQ2, we conducted an in-depth analysis to identify the similarities and differences between the two job profiles. It could be shown that the job profiles overlap in many points, but also show clear differences. There is an overlap in general AI skills, soft skills and IT skills (with the exception of programming languages). Differences are mainly in the programming languages and general IT skills. In summary, although the two job profiles overlap strongly, there are significant differences, and the standard job profile of an AI expert does not exist. There are various starting points for further research. A particularly interesting one is the development over time. The requirements of job profiles change over time, and it could be examined how dynamically new trends develop. In addition, the demand side was examined more closely in this paper. Further research could deal with how the supply side maps to this demand and whether and how differences in skills are distinct among potential specialists of CV engineers and NLP specialists. Furthermore, our 
analysis represents a starting point for further HR research, e.g., to investigate in detail how collaborative working in the context of $\mathrm{AI}$ is practiced in companies.

\section{References}

[1] Y. LeCun, Y. Bengio, and G. Hinton, "Deep learning”, Nature 521(7553), 2015, pp. 436-444.

[2] J. Yang, S. Li, Z. Wang, and G. Yang, "Real-Time Tiny Part Defect Detection System in Manufacturing Using Deep Learning”, IEEE Access 7, 2019, pp. 8927889291.

[3] O. Kwon, H.G. Kim, M.J. Ham, et al., "A deep neural network for classification of melt-pool images in metal additive manufacturing", Journal of Intelligent Manufacturing 31(2), 2020, pp. 375-386.

[4] X. Wan, L. Liu, S. Wang, and Y. Wang, "A Transfer Learning Strip Steel Surface Defect Recognition Network Based on VGG19”, In Y. Wang, K. Martinsen, T. Yu and K. Wang, eds., Advanced Manufacturing and Automation IX. Springer Singapore, Singapore, 2020, pp. 333-341.

[5] R. Yamashita, M. Nishio, R.K.G. Do, and K. Togashi, "Convolutional neural networks: an overview and application in radiology", Insights into Imaging 9(4), 2018, pp. 611-629.

[6] R. Miotto, F. Wang, S. Wang, X. Jiang, and J.T. Dudley, "Deep learning for healthcare: review, opportunities and challenges", Briefings in Bioinformatics 19(6), 2018, pp. 1236-1246.

[7] K. Fuchs, T. Grundmann, and E. Fleisch, "Towards Identification of Packaged Products via Computer Vision", Proceedings of the 9th International Conference on the Internet of Things - IoT 2019, ACM Press (2019), pp. 1-8.

[8] S. Chokkadi, S. MS, S. KB, and A. Bhandary, "A Study on various state of the art of the Art Face Recognition System using Deep Learning Techniques", International Journal of Advanced Trends in Computer Science and Engineering, 2019, pp. 1590-1600.

[9] Y. Guo, Y. Liu, A. Oerlemans, S. Lao, S. Wu, and M.S. Lew, "Deep learning for visual understanding: A review", Neurocomputing 187, 2016, pp. 27-48.

[10] I. Goodfellow, Y. Bengio, and A. Courville, Deep Learning, MIT Press, 2016.

[11] A. Krizhevsky, I. Sutskever, and G.E. Hinton, "ImageNet classification with deep convolutional neural networks", Communications of the ACM 60(6), 2012, pp. 84-90.

[12] H. Chen, R.H.L. Chiang, and V.C. Storey, "Business intelligence and analytics: From big data to big impact", MIS Quarterly: Management Information Systems 36(4), 2012, pp. 1165-1188.

[13] Z. Yang, D. Yang, C. Dyer, X. He, A. Smola, and E. Hovy, "Hierarchical Attention Networks for Document Classification", Proceedings of the 2016 Conference of the North American Chapter of the Association for Computational Linguistics: Human Language Technologies, Association for Computational Linguistics (2016), pp. 1480-1489.
[14] I.E. Fisher, M.R. Garnsey, and M.E. Hughes, "Natural Language Processing in Accounting, Auditing and Finance: A Synthesis of the Literature with a Roadmap for Future Research", Intelligent Systems in Accounting, Finance and Management 23(3), 2016, pp. $157-214$

[15] A., S., and D. John, "Survey on Chatbot Design Techniques in Speech Conversation Systems", International Journal of Advanced Computer Science and Applications 6(7), 2015.

[16] T. Wolf, V. Sanh, J. Chaumond, and C. Delangue, "TransferTransfo: A Transfer Learning Approach for Neural Network Based Conversational Agents", 2019.

[17] M. Adam, M. Wessel, and A. Benlian, "AI-based chatbots in customer service and their effects on user compliance", Electronic Markets, 2020.

[18] K. Mishev, A. Gjorgjevikj, I. Vodenska, L.T. Chitkushev, and D. Trajanov, "Evaluation of Sentiment Analysis in Finance: From Lexicons to Transformers", IEEE Access 8, 2020, pp. 131662-131682.

[19] A. Sciuto, A. Saini, J. Forlizzi, and J.I. Hong, "“Hey Alexa, What's Up?"”, Proceedings of the 2018 on Designing Interactive Systems Conference 2018 - DIS '18, ACM Press (2018), pp. 857-868.

[20] J.R. Bellegarda, "Spoken Language Understanding for Natural Interaction: The Siri Experience”, In Natural Interaction with Robots, Knowbots and Smartphones. Springer New York, New York, NY, 2014, pp. 3-14.

[21] H. Kortum, M. Leimkühler, and O. Thomas, "Leveraging Natural Language Processing to Analyze Scientific Content: Proposal of an NLP pipeline for the field of Computer Vision", Wirtschaftsinformatik 2021 Proceedings, 2021.

[22] L. Alekseeva, J. Azar, M. Gine, S. Samila, and B. Taska, "The demand for AI skills in the labor market", Labour Economics, 2021, pp. 102002.

[23] E. Anton, A. Behne, and F. Teuteberg, "THE HUMANS BEHIND ARTIFICIAL INTELLIGENCE - AN OPERATIONALISATION OF AI COMPETENCIES", Proceedings of the 28th European Conference on Information Systems (ECIS), An Online AIS Conference, June 15-17, 2020., (2020).

[24] D. Young, "The relative importance of technical and interpersonal skills for new information systems personnel", Journal of Computer Information Systems 36(4), 1996, pp. 66-71.

[25] P.H. Cheney, D.P. Hale, and G.M. Kasper, "Knowledge, skills and abilities of information systems professionals: past, present, and future", Information \& Management 19(4), 1990, pp. 237-247.

[26] S. Athey, and M. Wickham, "Required Skills for Information Systems Jobs in Australia", Journal of Computer Information Systems 36(2), 1996, pp. 60-63.

[27] C.D. Goldin, "Human capital", 2016.

[28] R. Gibbons, and M. Waldman, "Enriching a theory of wage and promotion dynamics inside firms", Journal of Labor Economics 24(1), 2006, pp. 59-107.

[29] R.S. Schuler, and I.C. MacMillan, "Gaining competitive advantage through human resource management practices", Human resource management 23(3), 1984, pp. 241-255. 
[30] R.K. Wagner, and R.J. Sternberg, "Practical intelligence in real-world pursuits: The role of tacit knowledge.", Journal of personality and social psychology 49(2), 1985, pp. 436.

[31] D. Joseph, S. Ang, R.H.L. Chang, and S.A. Slaughter, "Practical intelligence in IT: Assessing soft skills of IT professionals", Communications of the ACM 53(2), 2010, pp. 149-154.

[32] M.J. Stahl, and A.M. Harrell, "Evolution and validation of a behavioral decision theory measurement approach to achievement, power, and affiliation.”, Journal of Applied Psychology 67(6), 1982, pp. 744.

[33] W. Bauer, and C. Vocke, "Work in the Age of Artificial Intelligence - Challenges and Potentials for the Design of New Forms of Human-Machine Interaction", In 2020, pp. 493-501.

[34] J.K.-U. Brock, and F. von Wangenheim, "Demystifying AI: What Digital Transformation Leaders Can Teach You about Realistic Artificial Intelligence", California Management Review 61(4), 2019, pp. 110-134.

[35] A. Sulaiman, Y. Cheung, and C. Messom, "Artificial Intelligence Adoption: AI-readiness at Firm-Level”, PACIS 2018 Proceedings. 37., (2018).

[36] H.A.M. Hassan, G. Sansonetti, F. Gasparetti, and A. Micarelli, "Semantic-based tag recommendation in scientific bookmarking systems", RecSys '18: Twelfth ACM Conference on Recommender Systems, ACM (2018), pp. 465-469.

[37] Z. Almugbel, N. El, and N. Bugshan, "Automatic Structured Abstract for Research Papers Supported by Tabular Format using NLP", International Journal of Advanced Computer Science and Applications 10(2), 2019.

[38] M. Bilenko, and R.J. Mooney, "Adaptive duplicate detection using learnable string similarity measures", Proceedings of the ACM SIGKDD International Conference on Knowledge Discovery and Data Mining, ACM Press (2003), pp. 39-48.

[39] H. Hajishirzi, W.T. Yih, and A. Kołcz, "Adaptive nearduplicate detection via similarity learning", SIGIR 2010 Proceedings - 33rd Annual International ACM SIGIR Conference on Research and Development in Information Retrieval, ACM Press (2010), pp. 419-426.

[40] D. Nadeau, and S. Sekine, "A survey of named entity recognition and classification", Lingvisticae Investigationes. International Journal of Linguistics and Language Resources, 30(1), 2007, pp. 3-26.

[41] J. Li, A. Sun, J. Han, and C. Li, "A Survey on Deep Learning for Named Entity Recognition”, IEEE Transactions on Knowledge and Data Engineering, 2020, pp. 1-1.

[42] H.C. Wu, R.W.P. Luk, K.F. Wong, and K.L. Kwok, "Interpreting TF-IDF term weights as making relevance decisions", ACM Transactions on Information Systems 26(3), 2008, pp. 1-37.

[43] F. Wilcoxon, S.K. Katti, and R.A. Wilcox, "Critical values and probability levels for the Wilcoxon rank sum test and the Wilcoxon signed rank test", Selected tables in mathematical statistics 1, 1970, pp. 171-259.

[44] A. Zhang, "Peer assessment of soft skills and hard skills", Journal of Information Technology Education: Research 11(1), 2012, pp. 155-168.

[45] M.R. Weber, D.A. Finley, A. Crawford, and D. Rivera Jr, "An exploratory study identifying soft skill competencies in entry-level managers", Tourism and hospitality Research 9(4), 2009, pp. 353-361.

[46] J. Devlin, M.-W. Chang, K. Lee, and K. Toutanova, "BERT: Pre-training of Deep Bidirectional Transformers for Language Understanding”, 2018.

[47] M.E. Peters, M. Neumann, M. Iyyer, et al., "Deep contextualized word representations", 2018.

[48] J. Redmon, S. Divvala, R. Girshick, and A. Farhadi, "You Only Look Once: Unified, Real-Time Object Detection", arXiv:1506.02640 [cs], 2016.

[49] M.A.R. Ahad, S. Kobashi, and J.M.R.S. Tavares, "Advancements of Image Processing and Vision in Healthcare", Journal of Healthcare Engineering 2018, 2018, pp. 1-3.

[50] J. Gao, Y. Yang, P. Lin, and D.S. Park, "Computer Vision in Healthcare Applications", Journal of Healthcare Engineering 2018, 2018, pp. 1-4.

[51] A. Luckow, K. Kennedy, M. Ziolkowski, et al., "Artificial Intelligence and Deep Learning Applications for Automotive Manufacturing", 2018 IEEE International Conference on Big Data (Big Data), IEEE (2018), pp. 3144-3152.

[52] C. Friedman, and N. Elhadad, "Natural Language Processing in Health Care and Biomedicine", In Biomedical Informatics. Springer London, London, 2014, pp. 255-284.

[53] L. Robaldo, S. Villata, A. Wyner, and M. Grabmair, "Introduction for artificial intelligence and law: special issue "natural language processing for legal texts", Artificial Intelligence and Law 27(2), 2019, pp. 113115.

[54] D. Sarkar, Text analytics with Python: a practitioner's guide to natural language processing, Apress, 2019.

[55] L. Prechelt, “Are Scripting Languages Any Good? A Validation of Perl, Python, Rexx, and Tcl against C, C++, and Java", In 2003, pp. 205-270.

[56] X. Feng, Y. Jiang, X. Yang, M. Du, and X. Li, "Computer vision algorithms and hardware implementations: A survey", Integration 69, 2019, pp. 309-320.

[57] P. Mikalef, M.N. Giannakos, I.O. Pappas, and J. Krogstie, "The human side of big data: Understanding the skills of the data scientist in education and industry", IEEE Global Engineering Education Conference, EDUCON, IEEE Computer Society (2018), pp. 503512.

[58] M. Cubric, "Drivers, barriers and social considerations for AI adoption in business and management: A tertiary study", Technology in Society 62, 2020, pp. 101257. 